Article

\title{
Studies on 2-Arylhydrazononitriles: Synthesis of 3-Aryl-2- arylhydrazopropanenitriles and Their Utility as Precursors to 2-Substituted Indoles, 2-Substituted-1,2,3-Triazoles, and 1-Substituted Pyrazolo[4,3-d]pyrimidines
}

\author{
Khaled D. Khalil ${ }^{1,2, *}$ and Hamad M. Al-Matar ${ }^{1}$ \\ 1 Chemistry Department, Faculty of Science, University of Kuwait, P.O. Box 5969, \\ Safat 13060, Kuwait; E-Mail: h.almatar@ku.edu.kw \\ 2 Chemistry Department, Faculty of Science, Cairo University, Giza, 12613, Egypt \\ * Author to whom correspondence should be addressed; E-Mail: khd.khalil@yahoo.com; \\ Tel.: +965-2498-7559; Fax: +965-2481-6482.
}

Received: 27 August 2012; in revised form: 23 September 2012 / Accepted: 26 September 2012 / Published: 18 October 2012

\begin{abstract}
Coupling of 2-benzylmalononitrile with aromatic diazonium salts afforded 3-phenyl-2-arylhydrazonopropanenitriles $\mathbf{4 a}, \mathbf{b}$, which were rearranged into 2-cyanoindoles $\mathbf{5 a}, \mathbf{b}$ upon heating with $\mathrm{ZnCl}_{2}$ in the presence of glacial acetic acid. The produced indole derivatives 5a,b can be successfully used as valuable precursors to synthesize 1,2,4oxadiazolylindoles $\mathbf{8 a}, \mathbf{b}$. The reaction of arylhydrazononitriles $\mathbf{4 a}, \mathbf{b}$ with hydroxylamine afforded an amidoximes $\mathbf{9 a}, \mathbf{b}$ that could be cyclized into 1,2,3-triazole-4-amines 10a,b. In addition, 4a,b could be converted into 4-aminopyrazoles $\mathbf{1 2 a}, \mathbf{b}$ via condensation with chloroacetonitrile in the presence of triethylamine as a basic catalyst. Finally, compounds 12a,b were refluxed with dimethylformamide dimethylacetal (DMFDMA) to afford amidines 13a,b that were readily cyclized to the corresponding pyrazolo[4,3- $d]$ pyrimidines $\mathbf{1 4 a}, \mathbf{b}$ when refluxed with ammonium acetate.
\end{abstract}

Keywords: benzylidenemalononitrile; 2-arylhydrazononitrile; amidoxime; cyanoindole; dimethylformamide dimethylacetal; 1,2,3-triazole 


\section{Introduction}

2-Arylhydrazononitriles $\mathbf{4 a , b}$ are versatile reagents and their chemistry has recently attracted considerable interest [1-8]. In previous recent work we have established the utility of these compounds as precursors for 1,2,4-triazoles [5], 1,2,3-triazoles [6], and pyrazolo[1,5-a]pyrimidines [7,8]. In conjunction to that work we report herein an easy route to the title compounds and their utility as precursors for synthesis of various heterocycles. 1,2,3-Triazine derivatives are an important class of heterocyclic compounds that are considered useful precursors in organic synthesis and as pharmaceuticals (e.g., as antimalarials) [9-11]. In this article, we enabled development of an easy approach to 1,2,4-oxadiazolylindole [12,13], and pyrazolo[4,3- $d]$ pyrimidine derivatives of notable biological and pharmaceutical importance [14-16].

\section{Results and Discussion}

The hydrazononitriles $\mathbf{4 a , b}$ were synthesized by reducing benzylidenemalononitrile (1) with sodium borohydride as recently described, to yield $\mathbf{2}$ [17]. Coupling of compound $\mathbf{2}$ with aromatic diazonium salts afforded intermediates $\mathbf{3}$. It is believed that the initially formed $\mathbf{3 a}, \mathbf{b}$ readily undergo Japp-Klingmann cleavage [18] yielding the final isolable products $\mathbf{4 a , b}$ in $75 \%$, and $70 \%$ yield respectively. Compounds $\mathbf{4 a}, \mathbf{b}$ afforded the 2-cyanoindoles $\mathbf{5 a}, \mathbf{b}$ upon treatment with zinc chloride and glacial acetic acid. This is an example of the utility of the Fisher indole synthesis in the synthesis of 2-cyanoindoles (Scheme 1).

Scheme 1. Synthesis of indole-2-carbonitriles $\mathbf{5 a}, \mathbf{b}$.

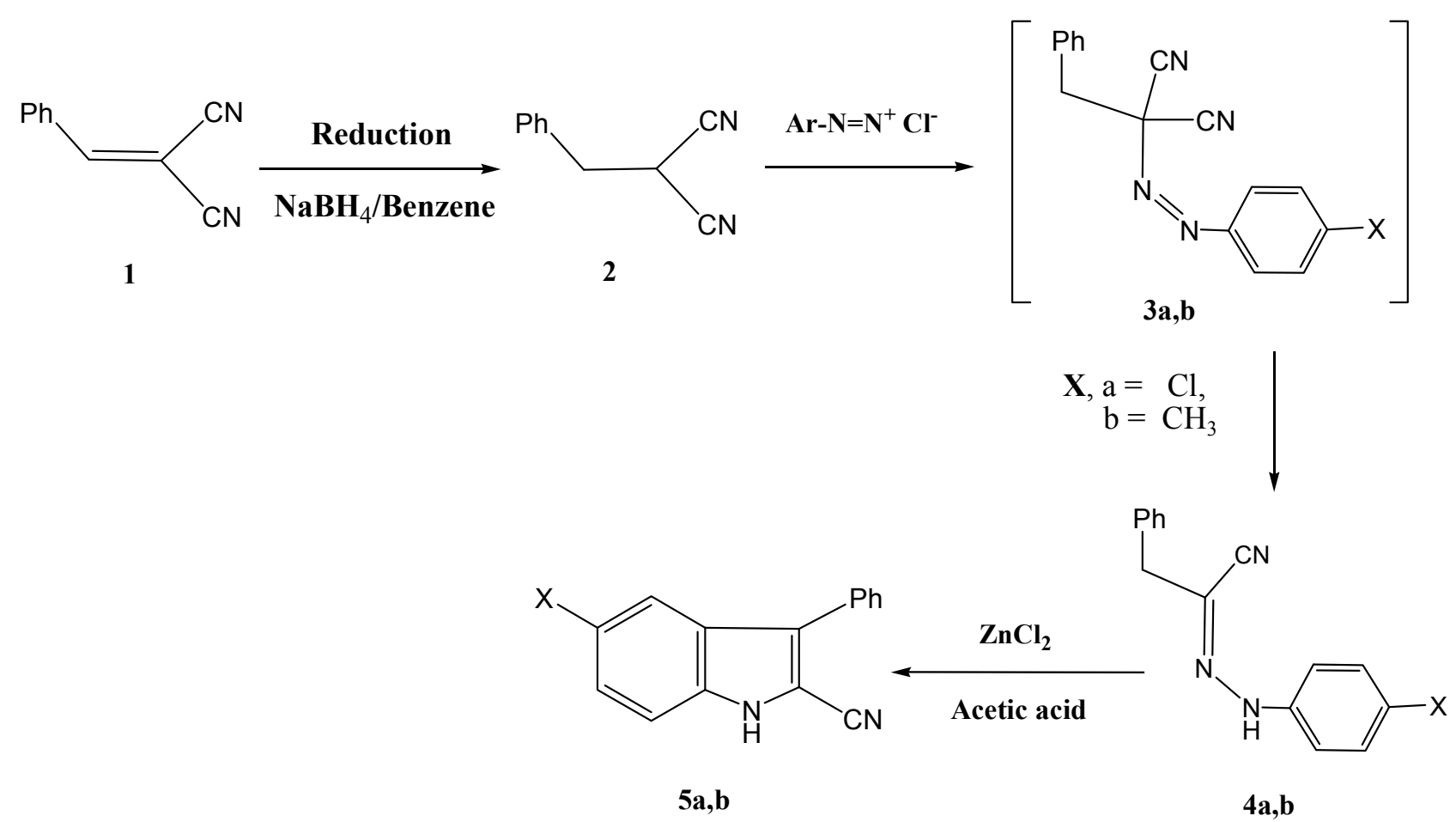

The 3-phenylindole-2-carbonitriles 5a,b reacted with hydroxylamine hydrochloride to yield amidoximes 6a,b. Reacting these products with dimethylformamide dimethylacetal (DMFDMA) 
afforded products $\mathbf{8 a}, \mathbf{b}$ in $68 \%$, and $65 \%$ yield respectively, rather than 7 , as indicated by a NOE experiment that showed an interaction between the indole- $\mathrm{H}-1$, at $10.4 \mathrm{ppm}$ and indole- $\mathrm{H}-7$, at 6.8-7.3 ppm (Scheme 2).

Scheme 2. Synthesis of 1,2,4-oxadiazolylindole derivatives 8a,b.

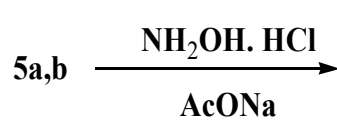<smiles>[X]c1ccc2[nH]c(/C(N)=N/O)c(-c3ccccc3)c2c1</smiles>

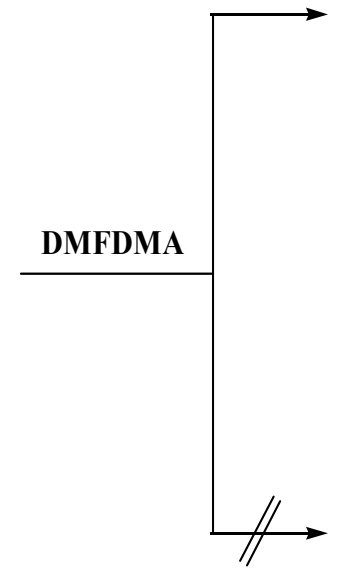<smiles>[X]c1ccc2[nH]c(-c3ncon3)c(-c3ccccc3)c2c1</smiles>

8a,b<smiles></smiles>

$7 \mathbf{a}, \mathbf{b}$

Our attention then shifted to explore the utility of 2-arylhydrazonals as efficient precursors to 1,2,3triazoles. Compounds $\mathbf{4 a , b}$ reacted with hydroxylamine hydrochloride to yield amidoximes $\mathbf{9 a , b}$ that could be cyclized into 10a,b or the isomeric 11a,b upon reflux in DMF. From the previously reported findings concerning this reaction, the structure of the product is not clear, where the 1,2,3-triazole 10a,b found a parallel in results reported for similar reactions under similar conditions [1-3]. Although cyclization into isoxazoles has been reported by either refluxing of amidoximes in acidic medium [4] or refluxing an ester derivative of an amidoxime in dimethylformamide [19], cyclization to a 1,2,4triazole via a Tiemann-like rearrangement has been reported by us in one case [5]. Structures 11a,b could be excluded due to the absence of any interaction between the $\mathrm{NH}_{2}$ protons and the aryl protons in a NOE experiment (Scheme 3). Moreover, we successfully confirmed that the correct structures are the 1,2,3-triazoles $\mathbf{1 0 a}, \mathbf{b}$ based on the obtained single crystal X-ray crystallography results recently reported by our group [6].

Compounds 4a,b was refluxed with chloroacetonitrile to yield 12a,b that were then refluxed with DMFDMA to give the expected amidines 13a,b. The amidines, so formed, were then cyclized in the presence of $\mathrm{NH}_{4} \mathrm{OAc}$ and glacial acetic acid to give pyrazolo[4,3- $\left.d\right]$ pyrimidines 14a,b (cf. Scheme 3). The structure of the products $\mathbf{1 4 a}, \mathbf{b}$ was confirmed by the spatial interaction between the $\mathrm{NH}_{2}$ protons, at $5.87 \mathrm{ppm}$, and aryl protons, at 7.08-7.17 ppm, in the NOE experiment. 
Scheme 3. Synthesis of 1,2,3-triazoles and pyrazolo[4,3- $d]$ pyrimidines.

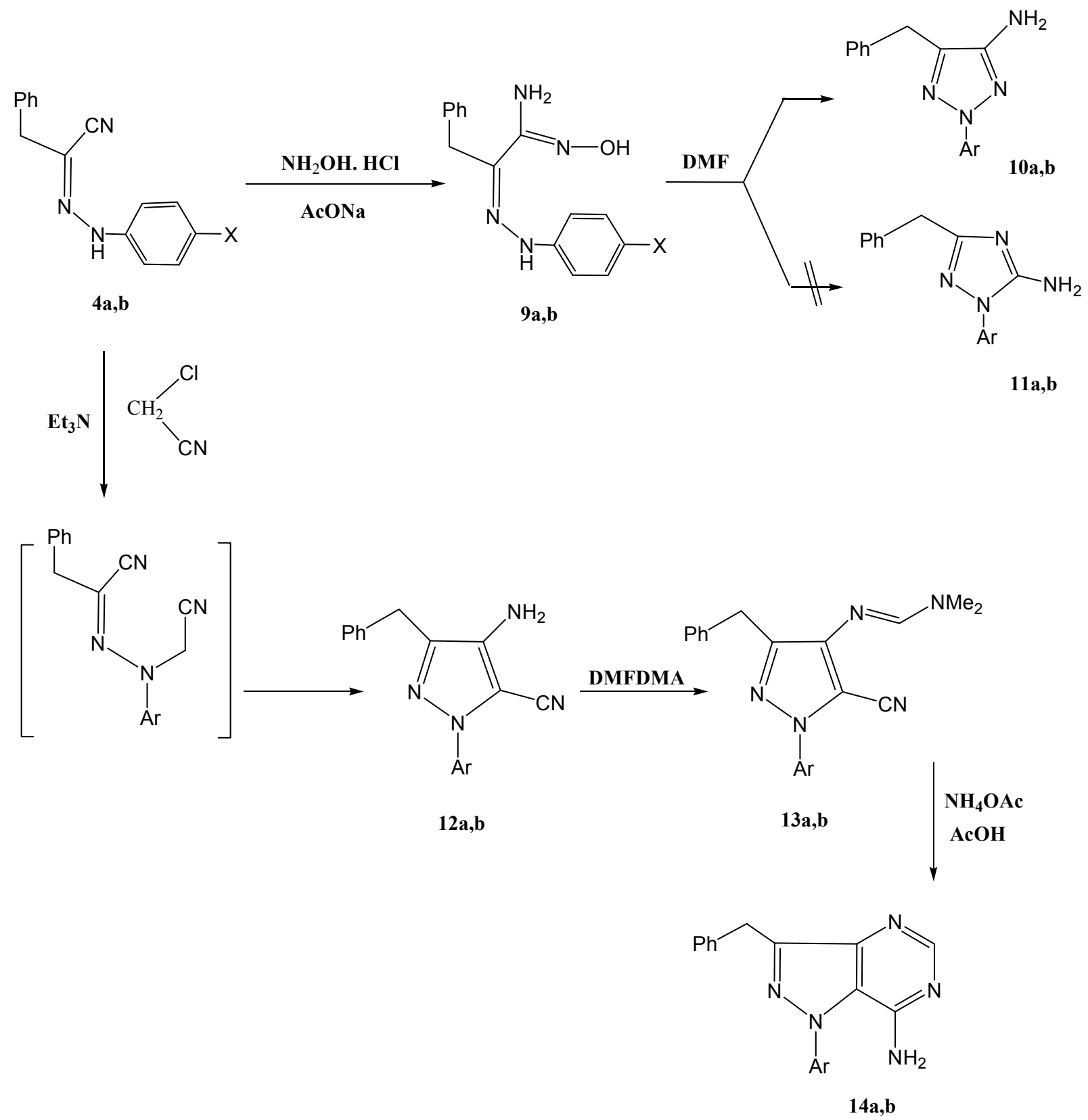

\section{Experimental}

\subsection{General Procedures}

Melting points were recorded on a Gallenkamp apparatus and are uncorrected. Infrared spectra (KBr) were determined on a Jasco FT/IR-6300 FT-IR instrument. NMR measurements were determined on a Bruker DPX spectrometer at $600 \mathrm{MHz}$ for ${ }^{1} \mathrm{H}-\mathrm{NMR}$ and $125 \mathrm{MHz}$ for ${ }^{13} \mathrm{C}-\mathrm{NMR}$, in DMSO- $d_{6}$ as solvent and using TMS as internal standard. Mass spectra were measured on GC MS DFS-hermo spectrometers. Elemental analyses were measured by means of an Elementar Vario Micro Cube. Microwave heating was carried out with a single mode cavity Explorer Microwave Synthesizer (CEM Corporation, NC, USA), producing continuous irradiation and equipped with simultaneous external air-cooling system. 


\subsection{Synthesis of 2-Benzylidenemalononitrile (2)}

This was prepared by the literature procedure [17]. A mixture of benzaldehyde (10 mmol) and malononitrile $(0.66 \mathrm{~g}, 10 \mathrm{mmol})$ was dissolved in aqueous ethanol $(1: 4,25 \mathrm{~mL})$ and stirred overnight. The reaction was followed to completion by TLC. To the pre-cooled reaction mixture, an equivalent amount of $\mathrm{NaBH}_{4}$ was added portionwise with stirring at $0{ }^{\circ} \mathrm{C}$ for $15 \mathrm{~min}$. The mixture was acidified with aqueous $\mathrm{HCl}$ and the product was extracted with $\mathrm{CH}_{2} \mathrm{Cl}_{2}$. The clear filtrate was evaporated under reduced pressure, and the remaining solid was collected by filtration. The solid product was then recrystallized from ethanol to give a colorless powder (82\%); mp $85-86{ }^{\circ} \mathrm{C}$ (lit. mp $86-88{ }^{\circ} \mathrm{C}$ [20]); IR $(\mathrm{KBr}): v=2188.4(\mathrm{CN}), 2198(\mathrm{CN}) \mathrm{cm}^{-1} ;{ }^{1} \mathrm{H}-\mathrm{NMR}: \delta=3.28(\mathrm{~d}, J=7.0 \mathrm{~Hz}, 2 \mathrm{H}), 3.88(\mathrm{t}, J=7.0 \mathrm{~Hz}$, 1H), 7.32-7.44 (m, 5H, phenyl); ${ }^{13} \mathrm{C}-\mathrm{NMR}: \delta=25.2(\mathrm{CH}), 37.1\left(\mathrm{CH}_{2}\right), 112.0(2 \mathrm{CN}), 128.6,129.0$, 129.2, 132.8 (aromatic carbons); MS, $m / z(\%): 156.07\left(\mathrm{M}^{+}, 100\right), 77$ (53); Anal. Calcd. for $\mathrm{C}_{10} \mathrm{H}_{8} \mathrm{~N}_{2}$ : C, 76.90; H, 5.16; N, 17.94. Found: C, 76.77; H, 5.09; N, 17.72.

Coupling of $\mathbf{2}$ with aryldiazonium chlorides. A cold solution of the appropriate aryldiazonium salt was prepared by adding sodium nitrite solution ( $1.4 \mathrm{~g}$ dissolved in $10 \mathrm{~mL}$ water) to a pre-cooled solution of the corresponding arylamine hydrochloride ( $p$-chloroaniline or $p$-toluidine, $10 \mathrm{mmol}$ of arylamine in $6 \mathrm{~mL} 6 \mathrm{M} \mathrm{HCl}$ ) with continuous stirring. The resulting aryldiazonium salt solutions were then added carefully to a cold ethanolic solution $(50 \mathrm{~mL})$ of benzylidenemalononitrile $(2,10 \mathrm{mmol})$ and sodium acetate trihydrate $(2.8 \mathrm{~g}, 20 \mathrm{mmol})$. The mixture was stirred at room temperature for $1 \mathrm{~h}$ and the solid product formed was collected by filtration, washed with water and recrystallized from ethanol.

2-[(4-Chlorophenyl)hydrazono]-3-phenylpropionitrile (4a). This compound was obtained as pale yellow solid (75\%); mp $\sim 148{ }^{\circ} \mathrm{C}$; IR (KBr): $v=3300$ (br. NH), $2185(\mathrm{CN}) \mathrm{cm}^{-1}$; ${ }^{1} \mathrm{H}-\mathrm{NMR}: \delta=2.61$ (s, 2H, $\left.\mathrm{CH}_{2}\right), 7.02$ (d, 2H, $J=8 \mathrm{~Hz}$, aryl), 7.34 (d, 2H, $J=8 \mathrm{~Hz}$, aryl), 7.44 (m, 5H, phenyl), 8.9 (s, 1H, $\mathrm{NH}) ;{ }^{13} \mathrm{C}-\mathrm{NMR}: \delta=30.2\left(\mathrm{CH}_{2}\right), 113.6,117.6(\mathrm{CN}), 119.5,125.3,126.8,128.0,129.8,133.0,135.9$ (aromatic carbons), $159.1(\mathrm{C}=\mathrm{N})$; $\mathrm{MS}, m / z(\%)$ : $269.1\left(\mathrm{M}^{+}, 100\right), 77(66)$; Anal. Calcd. for $\mathrm{C}_{15} \mathrm{H}_{12} \mathrm{ClN}_{3}$ : C, 66.79; H, 4.48; Cl, 13.14; N, 15.58. Found: C, 66.68; H, 4.40; Cl, 13.05; N, 15.46.

3-Phenyl-2-(p-tolylhydrazono)propionitrile (4b). This compound was obtained as a yellow solid (70\%); mp $\sim 126{ }^{\circ} \mathrm{C}$; IR (KBr): $v=3320$ (br. NH), $2189(\mathrm{CN}) \mathrm{cm}^{-1} ;{ }^{1} \mathrm{H}-\mathrm{NMR}: \delta=1.71\left(\mathrm{~s}, 3 \mathrm{H}, \mathrm{CH}_{3}\right)$, $2.66\left(\mathrm{~s}, 2 \mathrm{H}, \mathrm{CH}_{2}\right), 7.13$ (d, 2H, $J=8 \mathrm{~Hz}$, aryl), 7.18 (d, 2H, $J=8 \mathrm{~Hz}$, aryl), 7.22 (m, 5H, phenyl), 11.5 $(\mathrm{s}, 1 \mathrm{H}, \mathrm{NH}) ;{ }^{13} \mathrm{C}-\mathrm{NMR}: \delta=36.2\left(\mathrm{CH}_{3}\right), 38.6\left(\mathrm{CH}_{2}\right), 117.4(\mathrm{CN}), 118.9,123.5,127.3,129.2,132.4$, 134.6 (aromatic carbons), $157.5(\mathrm{C}=\mathrm{N})$; $\mathrm{MS}, \mathrm{m} / z(\%)$ : $249.31\left(\mathrm{M}^{+}, 100\right), 77$ (54). Anal. Calcd. for $\mathrm{C}_{16} \mathrm{H}_{15} \mathrm{~N}_{3}$ : C, 77.08; H, 6.06; N, 16.85. Found: C, 76.97; H, 5.98; N, 16.77.

Cyclization of $\mathbf{4 a}, \mathbf{b}$ in the presence of $\mathrm{ZnCl}_{2}$ and glacial acetic acid. A mixture of $\mathbf{4 a}, \mathbf{b}$ (10 mmol), zinc chloride $(1.34 \mathrm{~g}, 10 \mathrm{mmol})$, and glacial acetic acid $(50 \mathrm{~mL})$ was refluxed and followed by TLC till completion after $24 \mathrm{~h}$. The reaction mixture was poured into an ice/water mixture and the solid product, thus formed, was then collected by filtration and recrystallized from ethanol.

5-Chloro-3-phenyl-1H-indole-2-carbonitrile (5a). This compound was obtained as a yellow solid $(60 \%) ; \mathrm{mp} \sim 212{ }^{\circ} \mathrm{C}$; IR (KBr): $v=3300$ (br. NH), $2206(\mathrm{CN}) \mathrm{cm}^{-1} ;{ }^{1} \mathrm{H}-\mathrm{NMR}: \delta=7.03-7.26(\mathrm{~m}, 8 \mathrm{H}$, 
aryl \& phenyl), 11.1 (s, $1 \mathrm{H}, \mathrm{NH}) ;{ }^{13} \mathrm{C}-\mathrm{NMR}: \delta=117.6(\mathrm{CN}), 119.8,121.4,122.9,124.7,128.4,129.6$, 130.5, 133.0, 137.9 139.1, 142.2 (aromatic carbons); MS, m/z (\%): 252.05 ( $\left.\mathrm{M}^{+}, 100\right), 77$ (51); Anal. Calcd. for $\mathrm{C}_{15} \mathrm{H}_{9} \mathrm{ClN}_{2}$ : C, 71.29; H, 3.59; Cl, 14.03; N, 11.09. Found: C, 71.15; H, 3.52; Cl, 13.84; N, 10.97.

5-Methyl-3-phenyl-1H-indole-2-carbonitrile (5b). This compound was obtained as a colorless solid (70\%); mp $\sim 168{ }^{\circ} \mathrm{C}$; IR (KBr): $v=3320$ (br. NH), $2189(\mathrm{CN}) \mathrm{cm}^{-1} ;{ }^{1} \mathrm{H}-\mathrm{NMR}: \delta=1.81\left(\mathrm{~s}, 3 \mathrm{H}, \mathrm{CH}_{3}\right)$, 6.87-7.33 (m, 8H, phenyl), $10.8(\mathrm{~s}, 1 \mathrm{H}, \mathrm{NH}) ;{ }^{13} \mathrm{C}-\mathrm{NMR}: \delta=35.2\left(\mathrm{CH}_{3}\right), 117.6(\mathrm{CN}), 120.6,122.3,122.6$, 123.4, 127.0, 128.7, 129.4, 132.7, 132.8, 134.6, 139.8 (aromatic carbons); MS, $m / z$ (\%): $232.1\left(\mathrm{M}^{+}, 100\right)$, 77 (48); Anal. Calcd. for $\mathrm{C}_{16} \mathrm{H}_{12} \mathrm{~N}_{2}$ : C, 82.73; H, 5.21; N, 12.06. Found: C, 82.68; H, 5.14; N, 11.95.

\subsection{Synthesis of 1,2,4-Oxadiazolyl-indoles $\mathbf{8 a}, \mathbf{b}$}

A mixture of 5a,b $(10 \mathrm{mmol})$, hydroxylamine hydrochloride $(0.69 \mathrm{~g}, 10 \mathrm{mmol})$, and sodium acetate ( $3 \mathrm{~g}, 25 \mathrm{mmol})$ in ethanol $(25 \mathrm{~mL})$ was refluxed for $5 \mathrm{~h}$. The reaction mixture was poured into ice/water with stirring while a yellow solid separated and was then collected by filtration. The crude product was refluxed with DMFDMA for 6 h. The pure products 8a,b were purified by recrystallization from ethanol.

3-(5-Chloro-3-phenyl-1H-indol-2-yl)-1,2,4-oxadiazole (8a). Obtained as a pale yellow powder (68\%); $\mathrm{mp} \sim 142{ }^{\circ} \mathrm{C}$; IR (KBr): $v=1586$ (aromatic $\mathrm{C}=\mathrm{C}$ ) $\mathrm{cm}^{-1} ;{ }^{1} \mathrm{H}-\mathrm{NMR}: \delta=6.84-7.31$ (m, 9H, aromatic), 10.4 (s, 1H, NH, imidazole); ${ }^{13} \mathrm{C}-\mathrm{NMR}: \delta=112.3,115.2,121.2,122.8,123.4,124.0,127.9,128.5$, 129.1, 131.4, 133.8, 134.7, 137.9, 148.6 (aromatic carbons); MS, $m / z$ (\%): $295.1\left(\mathrm{M}^{+}, 56\right), 77$ (100); Anal. Calcd. for $\mathrm{C}_{16} \mathrm{H}_{10} \mathrm{ClN}_{3} \mathrm{O}$ : C, 64.98; H, 3.41; Cl, 11.99; N, 14.21. Found: C, 64.91; H, 3.36; $\mathrm{Cl}, 11.91 ; \mathrm{N}, 14.13$.

3-(5-Methyl-3-phenyl-1H-indol-2-yl)-1,2,4-oxadiazole (8b). Obtained as a yellow solid (65\%); $\mathrm{mp} \sim 124{ }^{\circ} \mathrm{C}$; IR (KBr): $v=3100$ (aromatic $\left.\mathrm{CH}\right) \mathrm{cm}^{-1} ;{ }^{1} \mathrm{H}-\mathrm{NMR}: \delta=2.69\left(\mathrm{~s}, 3 \mathrm{H}, \mathrm{CH}_{3}\right), 6.72-7.28(\mathrm{~m}$, 9H, aromatic), 10.1 (s, 1H, NH, imidazole); ${ }^{13} \mathrm{C}-\mathrm{NMR}: \delta=36.3\left(\mathrm{CH}_{3}\right), 110.6,112.7,119.3,121.7$. 122.6, 122.9, 123.9, 127.4, 128.9, 129.6, 131.2, 132.6, 134.8142 .6 (aromatic carbons); $\mathrm{MS}, \mathrm{m} / z(\%)$ : $275.1\left(\mathrm{M}^{+}, 83\right), 77$ (100); Anal. Calcd. for $\mathrm{C}_{17} \mathrm{H}_{13} \mathrm{~N}_{3} \mathrm{O}$ : C, 74.17; H, 4.76; N, 15.26. Found: C, 74.09; $\mathrm{H}, 4.66 ; \mathrm{N}, 15.13$.

\subsection{Synthesis of 1,2,3-Triazole Derivatives 10a,b}

A mixture of 4a,b (10 mmol), hydroxylamine hydrochloride $(0.69 \mathrm{~g}, 10 \mathrm{mmol})$, and sodium acetate ( $3 \mathrm{~g}, 25 \mathrm{mmol})$ was dissolved in ethanol $(25 \mathrm{~mL})$. The mixture was refluxed for $4 \mathrm{~h}$. The reaction mixture was poured into ice/water with stirring while a yellow solid separated and was then collected by filtration. The crude product, so formed, was then refluxed in DMF for $5 \mathrm{~h}$ and the reaction mixture was poured into cold water. The products $\mathbf{1 0 a}, \mathbf{b}$ were purified by crystallization from ethanol.

5-Benzyl-2-(4-chlorophenyl)-2H-1,2,3-triazol-4-amine (10a). It was obtained as a yellow solid (75\%); $\mathrm{mp}>250{ }^{\circ} \mathrm{C}$; IR ( $\left.\mathrm{KBr}\right): v=3330$ (br. $\left.\mathrm{NH}_{2}\right) \mathrm{cm}^{-1} ;{ }^{1} \mathrm{H}-\mathrm{NMR}: \delta=3.65\left(\mathrm{~s}, 2 \mathrm{H}, \mathrm{CH}_{2}\right), 6.87\left(\mathrm{~s}, 2 \mathrm{H}, \mathrm{NH}_{2}\right)$, 7.01-7.23 (m, 9H, aromatic); ${ }^{13} \mathrm{C}-\mathrm{NMR}: \delta=33.1\left(\mathrm{CH}_{2}\right), 104.8,121.3,122.7,122.9,125.7,128.3$, 
129.1, 132.0, 134.6, 141.0 (aromatic carbon); MS, $m / z$ (\%): $284.08\left(\mathrm{M}^{+}, 65\right), 77$ (84); Anal. Calcd. for $\mathrm{C}_{15} \mathrm{H}_{13} \mathrm{ClN}_{4}$ : C, 63.27; H, 4.60; Cl, 12.45; N, 19.68. Found: C, 63.18; H, 4.53; Cl, 12.34; N, 19.62.

5-Benzyl-2-(4-tolyl)-2H-1,2,3-triazol-4-amine (10b). It was obtained as a yellow solid (70\%); $\mathrm{mp} \sim 197{ }^{\circ} \mathrm{C}$; IR (KBr): $v=3340$ (br. $\left.\mathrm{NH}_{2}\right) \mathrm{cm}^{-1} ;{ }^{1} \mathrm{H}-\mathrm{NMR}: \delta=2.44\left(\mathrm{~s}, 3 \mathrm{H}, \mathrm{CH}_{3}\right), 3.61\left(\mathrm{~s}, 2 \mathrm{H}, \mathrm{CH}_{2}\right)$, $5.82\left(\mathrm{~s}, 2 \mathrm{H}, \mathrm{NH}_{2}\right), 7.01-7.24$ (m, 9H, aromatic); ${ }^{13} \mathrm{C}-\mathrm{NMR}: \delta=30.8\left(\mathrm{CH}_{3}\right), 32.6\left(\mathrm{CH}_{2}\right), 106.4,119.6$, 121.2, 122.7, 124.3, 128.1, 128.4, 131.8, 133.2, 139.1 (aromatic carbons); $\mathrm{MS}, m / z(\%): 264.14\left(\mathrm{M}^{+}, 46\right)$, 77 (100); Anal. Calcd. for $\mathrm{C}_{16} \mathrm{H}_{16} \mathrm{~N}_{4}$ : C, 72.70; H, 6.10; N, 21.20. Found: C, 72.57; H, 6.02; N, 21.13.

\subsection{Cyclization of $\mathbf{4 a , b}$ with Chloroacetonitrile in the Presence of $E t_{3} N$}

A mixture of $4 \mathbf{a}, \mathbf{b}(10 \mathrm{mmol})$, chloroacetonitrile $(0.75 \mathrm{~g}, 10 \mathrm{mmol})$, and triethylamine $(0.5 \mathrm{~mL})$ was irradiated at $80 \mathrm{~W}$ for $5 \mathrm{~min}$ (final temperature $140{ }^{\circ} \mathrm{C}$ ). The reaction mixture was poured into a $\mathrm{HCl} /$ water mixture and the solid product, so formed, was then collected by filtration and recrystallized from ethanol.

4-Amino-3-benzyl-1-(4-chlorophenyl)-1H-pyrazole-5-carbonitrile (12a). This compound was obtained as a yellow solid (67\%); $\mathrm{mp} \sim 227{ }^{\circ} \mathrm{C}$; IR (KBr): $v=3350$ (br. $\left.\mathrm{NH}_{2}\right), 2210(\mathrm{CN}) \mathrm{cm}^{-1}$; ${ }^{1} \mathrm{H}-\mathrm{NMR}$ : $\delta=3.86\left(\mathrm{~s}, 2 \mathrm{H}, \mathrm{CH}_{2}\right), 6.87\left(\mathrm{~s}, 2 \mathrm{H}, \mathrm{NH}_{2}\right), 7.01-7.26\left(\mathrm{~m}, 9 \mathrm{H}\right.$, aromatic); ${ }^{13} \mathrm{C}-\mathrm{NMR}: \delta=31.6\left(\mathrm{CH}_{2}\right)$, $117.8(\mathrm{CN}), 119.9,121.5,122.3,125.1,125.4,127.2,129.8,132.6,133.8,135.1,138.7$ (aromatic carbons); MS, $m / z(\%): 308.08\left(\mathrm{M}^{+}, 27\right), 77$ (100); Anal. Calcd. for $\mathrm{C}_{17} \mathrm{H}_{13} \mathrm{ClN}_{4}$ : C, 66.13; H, 4.24; $\mathrm{Cl}$, $11.48 ; \mathrm{N}, 18.15$. Found: C, 66.04; H, 4.07; Cl, 11.33; N, 18.05.

4-Amino-3-benzyl-1-p-tolyl-1H-pyrazole-5-carbonitrile (12b). This compound was obtained as a yellow solid (70\%); $\mathrm{mp} \sim 204{ }^{\circ} \mathrm{C}$; IR (KBr): $v=3330$ (br. $\left.\mathrm{NH}_{2}\right), 2190(\mathrm{CN}) \mathrm{cm}^{-1} ;{ }^{1} \mathrm{H}-\mathrm{NMR}: \delta=2.27$ $\left(\mathrm{s}, 3 \mathrm{H}, \mathrm{CH}_{3}\right), 3.62\left(\mathrm{~s}, 2 \mathrm{H}, \mathrm{CH}_{2}\right), 6.41\left(\mathrm{~s}, 2 \mathrm{H}, \mathrm{NH}_{2}\right), 6.94-7.21\left(\mathrm{~m}, 9 \mathrm{H}\right.$, aromatic); ${ }^{13} \mathrm{C}-\mathrm{NMR}: \delta=35.9$ $\left(\mathrm{CH}_{3}\right), 38.1\left(\mathrm{CH}_{2}\right), 117.7(\mathrm{CN}), 119.2,121.1,121.7,124.2,127.9,128.6,129.7,131.2,132.4,134.0$, 136.4 (aromatic carbons); MS, $m / z(\%)$ : $288.14\left(\mathrm{M}^{+}, 62\right), 77$ (100); Anal. Calcd. for $\mathrm{C}_{18} \mathrm{H}_{16} \mathrm{~N}_{4}$ : C, 74.98; H, 5.59; N, 19.43. Found: C, 74.88; H, 5.47; N, 19.27.

\subsection{Synthesis of Pyrazolopyrimidine Derivatives}

A mixture of 12a,b (10 mmol) and dimethylformamide dimethylacetal $(1.8 \mathrm{~g}, 15 \mathrm{mmol})$ in dry xylene $(50 \mathrm{~mL})$ was refluxed for $6 \mathrm{~h}$. The reaction mixture was cooled and then the product, so formed, was refluxed with ammonium acetate $(1.54 \mathrm{~g}, 20 \mathrm{mmol})$ and glacial acetic acid $(25 \mathrm{~mL})$ for $4 \mathrm{~h}$. The reaction mixture was cooled and treated with petroleum ether whereby a yellowish solid precipitated and was collected by filtration. The pure product was obtained by crystallized from ethanol.

3-Benzyl-1-(4-chlorophenyl)-1H-pyrazolo[4,3-d]pyrimidin-7-amine (14a). This compound was obtained as a yellow solid (72\%); mp $>250{ }^{\circ} \mathrm{C}$; IR (KBr): $v=3350\left(\right.$ br. $\left.\mathrm{NH}_{2}\right) \mathrm{cm}^{-1} ;{ }^{1} \mathrm{H}-\mathrm{NMR}: \delta=3.36$ $\left(\mathrm{s}, 2 \mathrm{H}, \mathrm{CH}_{2}\right), 5.87\left(\mathrm{~s}, 2 \mathrm{H}, \mathrm{NH}_{2}\right), 7.08-7.17$ (m, 9H, aromatic), 8.98 (s, 1H, CH pyrimidine); ${ }^{13} \mathrm{C}-\mathrm{NMR}$ : $\delta=34.6\left(\mathrm{CH}_{2}\right), 104.7,110.4,114.0,119.1,121.4,123.9,124.2,127.3,128.7,130.2,131.7,134.3$, 139.7 (aromatic carbons); MS, $m / z(\%): 335.09\left(\mathrm{M}^{+}, 48\right), 77(100)$; Anal. Calcd. for $\mathrm{C}_{18} \mathrm{H}_{14} \mathrm{ClN}_{5}$ : $\mathrm{C}$, 64.38; H, 4.20; Cl, 10.56; N, 20.86. Found: C, 64.26; H, 4.06; Cl, 10.39; N, 20.67. 
3-Benzyl-1-p-tolyl-1H-pyrazolo[4,3-d]pyrimidin-7-amine (14b). This compound was obtained as a yellow solid (65\%); mp $>250{ }^{\circ} \mathrm{C}$; IR (KBr): $v=3330$ (br. $\left.\mathrm{NH}_{2}\right) \mathrm{cm}^{-1} ;{ }^{1} \mathrm{H}-\mathrm{NMR}: \delta=2.84\left(\mathrm{~s}, 3 \mathrm{H}, \mathrm{CH}_{3}\right)$, $3.17\left(\mathrm{~s}, 2 \mathrm{H}, \mathrm{CH}_{2}\right), 5.61\left(\mathrm{~s}, 2 \mathrm{H}, \mathrm{NH}_{2}\right), 6.86-7.19$ (m, 9H, aromatic), 8.62 (s, 1H, CH pyrimidine); ${ }^{13} \mathrm{C}-\mathrm{NMR}: \delta=36.4\left(\mathrm{CH}_{3}\right), 38.6\left(\mathrm{CH}_{2}\right), 105.2,112.4,119.1,119.7,122.1,124.9,128.2,128.6,130.9$, 132.3, 134.6, 135.0, 137.8 (aromatic carbons); MS, $m / z(\%)$ : $315.1\left(\mathrm{M}^{+}, 53\right), 77$ (100); Anal. Calcd. for $\mathrm{C}_{19} \mathrm{H}_{17} \mathrm{~N}_{5}$ : C, 72.36; H, 5.43; N, 22.21. Found: C, 72.25; H, 5.31; N, 22.08.

\section{Conclusions}

2-Arylhydrazono-3-propanenitriles are readily obtainable versatile intermediates in the syntheses of a diversity of heterocycles, especially indoles, pyrazoles, 1,2,3-triazole, and pyrazolo[4,3-d]pyrimidines, thus proving the general scope of our newly reported findings on the reactions of 2-aryl-hydrazononitriles.

\section{Acknowledgments}

Support for this work received from the University of Kuwait through a research grant (SC09/07) and the facilities of Analab/SAF by research grants (GC01/01, GC01/05, GC01/03 and GS03/01) are gratefully acknowledged. The authors would like to thank M. Elnagdi for his valuable contributions.

\section{References}

1. El-Dusouqui, O.M.E.; Abdelkhalik, M.M.; Al-Awadi, N.A.; Dib, H.H.; George, B.J.; Elnagdi, M.H. Chemistry of 2-arylhydrazonals: Utility of substituted 2-arylhydrazono-3oxoalkanals as precursors for 3-oxoalkanonitriles, 3-aminoisoxazole and 1,2,3- and 1,2,4-triazoles. J. Chem. Res. 2006, 5, 295-302.

2. Ghozlan, S.A.S.; Abdelhamid, I.A.; Ibrahem, H.M.; Elnagdi, M.H. Studies with 2-arylhydrazononitriles: A new convenient synthesis of 2,4-disubstituted-1,2,3-triazole-5-amines. ARKIVOC 2006, 15, 53-60.

3. Behbehani, H.; Ibrahim, H.M.; Makhseed, S. Studies with 3-oxoalkanonitriles: Synthesis and reactivity of 3-oxo-3-(1-methylindolyl)propanenitrile. Heterocycles 2009, 78, 3081-3090.

4. Elnagdi, M.H.; Elmoghayar, M.R.H.; Hafez, E.A.A.; Alnima, H.H. Reaction of 2-arylhydrazono3-oxonitriles with hydroxylamine. Synthesis of 3-amino-4-arylazoisoxazoles. J. Org. Chem. 1975, 40, 2604-2607.

5. Al-Matar, H.M.; Riyadh, S.M.; Elnagdi, M.H. 2-Arylhydrazononitriles in heterocyclic synthesis: A novel route to 1,3-diaryl-1,2,4-triazol-5-amines via a Tiemann rearrangement of Arylhydrazonoamidoximes. ARKIVOC 2007, 13, 53-62.

6. Al-Matar, H.M.; Adam, A.Y.; Khalil, K.D.; Elnagdi, M.H., Studies with 3-oxoalkanenitriles: Novel rearrangements observed while exploring the utility of 2-cyanoacetyl-1-methylpyrrole as a precursor to pyrrole substituted heterocyclic compounds. ARKIVOC 2012, 6, 1-15.

7. Al-Matar, H.M.; Khalil, K.D.; Al-Dorri, D.M.; Elnagdi, M.H. Efficient routes to pyrazolo[3,4e][1,2,4]triazines and a new ring system: $[1,2,4]$ triazino[5,6-d][1,2,3]triazines. Molecules 2010, $15,3302-3310$. 
8. Riyadh, S.M.; Al-Matar, H.M.; Elnagdi, M.H. Studies with 2-arylhydrazono-nitriles: Further investigations on reactivity of 2-arylhydrazononitriles towards hydroxylamine. J. Heterocycl. Chem. 2008, 45, 975-979.

9. Stevens, M.F.G. The medicinal chemistry of 1,2,3-triazines. Prog. Med. Chem. 1976, 13, 205-269.

10. Curd, F.H.S.; Landquist, J.K.; Rose, F.L. Synthetic antimalarials. Part XII. Some 1:3:5-triazine derivatives. J. Chem. Soc. 1947, 154-160.

11. Migawa, M.T.; Drach, J.C.; Townsend, L.B. Design, synthesis and antiviral activity of novel 4,5disubstituted 7-( $\beta$-D-ribofuranosyl)pyrrolo[2,3-d][1,2,3]triazines and the novel 3-amino-5-methyl1-( $\beta$-D-ribofuranosyl)- and 3-amino-5-methyl-1-(2-deoxy- $\beta$-D-ribofuranosyl)-1,5-dihydro-1,4,5,6,7,8hexaazaacenaphthylene as analogues of triciribine. J. Med. Chem. 2005, 48, 3840-3851.

12. Alyab'ev, S.B.; Kravchenko, D.V.; Ivashchenko, A.V. Functionalization of oxadiazolylindole systems. Russ. J. Org. Chem. 2009, 45, 719-724.

13. Pal, M.; Sharma, N.K.; Priyanka, J.K.K. Synthetic and biological multiplicity of isatin: A review. J. Adv. Sci. Res. 2011, 2, 35-44.

14. Masevicius, V.; Juskenas, R.; Tumkevicius, S. Synthesis of a novel heterocyclic system-pyrazolo[5,4,3-de]pyrimido-[4,5-e][1,4]diazepine. Chem. Heterocycl. Compd. 2007, 43, $1593-1594$.

15. Sroka, I.M.; Heiss, E.H.; Havlicek, L.; Totzke, F.; Aristei, Y.; Pechan, P.; Kubbutat, M.H.G.; Strnad, M.; Dirsch, V.M. A novel roscovitine derivative potently induces G1-phase arrest in platelet-derived growth factor-BB-activated vascular smooth muscle cells. Mol. Pharmacol. 2010, 77, 255-261.

16. Fevig, J.M.; Cacciola, J.; Buriak, J.; Rossi, K.A.; Knabb, R.M.; Luettgen, J.M.; Wong, P.C.; Bai, S.A.; Wexler, R.R.; Lam, P.Y.S. Preparation of 1-(4-methoxyphenyl)-1H-pyrazolo[4,3d]pyrimidin-7(6H)-ones as potent, selective and bioavailable inhibitors of coagulation factor Xa. Bioorg. Med. Chem. Lett. 2006, 16, 3755-3760.

17. Tayyari, F.; Wood, D.E.; Fanwick, P.E.; Sammelson, R.E. Monosubstituted Malononitriles: Efficient one-pot reductive alkylation of malononitrile with aromatic aldehydes. Synthesis 2008, 279-285.

18. Severin, T.; Poehlmann, H. Umsetzungen mit Monohydrazonen von Dicarbonylverbindungen, VI: Synthesen von 4-Oxocarbonsäuren und deren Estern mit Hilfe von $\alpha$-Hydrazonoaldehyden. Chem. Ber. 1978, 111, 1564-1577.

19. Ghozlan, S.A.S.; Badahdah, K.O.; Abdelhamid, I.A. An easy synthesis of 5-functionally substituted ethyl 4-amino-1-aryl- pyrazolo-3-carboxylates: Interesting precursors to sildenafil analogues. Beilstein J. Org. Chem. 2007, 3, 1-3.

20. Katritzky, A.R.; Akue-Gedu, R.; Vakulenko, A.V. C-cyanation with 1-cyanobenzotriazole. ARKIVOC 2007, 3, 5-12.

Sample Availability: Samples of the compounds 5, 8, 10, and 14 are available from the authors.

(C) 2012 by the authors; licensee MDPI, Basel, Switzerland. This article is an open access article distributed under the terms and conditions of the Creative Commons Attribution license (http://creativecommons.org/licenses/by/3.0/). 